\title{
Tuberculosis of Spine: Current Views in Diagnosis and Management
}

\author{
Myung-Sang Moon ${ }^{1,2}$ \\ ${ }^{I}$ Department of Orthopedic Surgery, Cheju Halla General Hospital, Jeju, Korea \\ ${ }^{2}$ Moon-Kim's Institute of Orthopedic Research, Seoul, Korea
}

Tuberculosis is the chronic consumptive disease and currently the world's leading cause of death. Tuberculous spondylitis is a less common yet the most dangerous form of skeletal tuberculosis. The recent re-emergence of Mycobacterium tuberculosis ( $M$. tuberculosis) hints at a possible resurgence of tuberculosis in the coming years. This article discusses the clinical manifestations, diagnosis and treatment of tuberculous spondylitis, and updates material that the author has previously published on the subject. Treatment should be individualized according to different indications which is essential to recovery. A treatment model is suggested on the basis of the author's vast personal experiences.

Keywords: Tuberculous spondylitis; Mycobacterium tuberculosis

\section{Introduction}

Tuberculosis in human is usually a chronic disease with weight loss, and currently the world's leading cause of death [1-5]. Tuberculous spondylitis is a less common yet the most dangerous form of skeletal tuberculosis [6-10]. The recent re-emergence of Mycobacterium tuberculosis (M. tuberculosis) hints at a possible resurgence of tuberculosis in the coming years [1-3,5]. This article discusses the clinical manifestations, diagnosis and medical and surgical treatments of tuberculous spondylitis, and updates material that the author has previously published on the subject. Individualizing the treatment according to different indications is essential to recovery [5]. A treatment model is suggested, which may help to improve treatment outcomes and reduce complications.

\section{Tuberculosis: The Deadliest Infection}

Approximately 8 million tuberculosis cases have been identified worldwide, which have resulted in 2 million deaths [1-3,5]. Ninety-five percent of tuberculosis patients are in the developing regions of the world. According to the World Health Organization, tuberculosis causes 1.81 million deaths in Asia each year [1,2]. China has 1.4 million new cases every year [3]. In some African countries (East and Central Africa), the number of reported tuberculosis cases has doubled or even tripled with the spread of human immunodeficiency virus and acquired immunodeficiency syndrome (HIV/AIDs) [1,2]. In HIVnegative patients, around $3 \%$ to $5 \%$ of tuberculosis is skeletal, whereas in HIV-positive patients, about $60 \%$ of the cases involves the bone. The emergence of AIDs has also significantly increased the incidence of disseminated Mycobacterium avium complex (MAC) infection, which

Received Mar 2, 2009; Revised Oct 19, 2013; Accepted Oct 19, 2013

Corresponding author: Myung-Sang Moon

Moon-Kim's Institute of Orthopedic Research,

Shi-bum Apt. 14-105, 45, 63-ro, Yeongdeungpo-gu, Seoul 150-761, Korea

Tel: +82-2-780-5387, E-mail: msmoonos@hotmail.com 
occurs almost exclusively in patients with a CD4 lymphocyte count below 100 cells/mm [2,3,5]. Apart from the spread of HIV/AIDs, recent resurgence of chemoresistant variants of $M$. tuberculosis in particular poses further threat, and has raised great concerns worldwide. Taken together, the data indicate a need to prepare for a re-emergence of skeletal tuberculosis in the coming years $[2,5]$.

The spine is involved in 50\% of osteoarticular tuberculosis cases. Spinal tuberculosis is the form most dangerous, as it may cause bone destruction of the vertebral body, spinal deformity and/or paraplegia and pulmonary insufficiency secondary to the deformity of thoracic cage. When the body of the spine and the neural arch are involved, particularly in children, translational instability of the affected spine with anterior or lateral vertebral displacement may occur, with the resultant deformity posing a risk of cord compression to the patient $[11,12]$.

In children with Pott's disease, the risk of late kyphosis depends on the age ( $<5$ years), level of involvement (thoracic), extent of the disease ( $>2$ vertebrae), endplate damages and the pattern of involvement. Dislocation of facets, posterior retropulsion of the diseased fragment and toppling of the superior vertebra suggest the worst prognosis for kyphosis $[11,12]$. Buckling (invaginating angulatory) collapse of the spine is unique to childhood spinal tuberculosis at the lower dorsal and dorsolumbar spine. Fortunately, the above risk factors have not been seen in early spinal tuberculosis. However, in adults, when the lumbar spine is involved, the pattern of vertebral collapse is different from that of the dorsolumbar spine, and is telescoping (non-kyphotic vertebral body collapse) in nature [13]. It is strongly advised that kyphosis of more than $45^{\circ}$ not be allowed, as such puts the posterior spinal muscles at a mechanical disadvantage, adding to the deforming force. It contributes not only to a progression of kyphosis, but also to the occurrence of paraplegia [14]. Prophylactic stabilization procedures should therefore definitely be considered to prevent further progression of kyphosis.

\section{Immunity and Tuberculosis}

Delays in presentation and diagnosis are almost universal in the treatment of spinal infection. Immunosuppressed patients tend to have longer delays to presentation and diagnosis due to less vigorous inflammation and pain generation [5]. Therefore, it is important to know the patient's immune state in the HIV/AIDs and substance abuse era, because immunosuppression is closely linked with the infection [15-21].

Humoral immunity requires proper functioning and quantity of complement proteins and immunoglobulins in addition to antigen presentation cells, while cellular immunity is delivered by the direct action of $\mathrm{B}$ and $\mathrm{T}$ lymphocytes and antigen phagocytes such as macrophages.

To evaluate the patient's pre-therapy physical condition, the predisposing factors of skeletal tuberculosis as a firststep measure should be considered. The factors include age (the elderly), malnutrition, agammaglobulinemia, diabetes mellitus, HIV infection, malignancy, renal failure, substance abuse, long-term use of anti-rheumatic agents (steroids, anti-tumour necrosis factor [TNF]- $\alpha$ blockers and B-cell depleting therapeutic agents, methotrexate) and anti-cancer chemotherapeutic agents [20,21]. Tuberculosis is a potentially fatal complication of immunosuppressive therapy.

Immunity in tuberculosis being a purely cell-mediated defense, lymphoproliferative response to antigen or mitogen stimulation has been widely used as in vitro correlate of cell-mediated immunity. Particularly T-cell mediated cellular immunity has been suggested to be important in mycobacterial infection. Also it is known that there is an imbalance between helper $(\mathrm{H})$ and suppressor $(\mathrm{S}) \mathrm{T}$ cells in the pathogenesis of tuberculosis in human. H/S ratio in extrapulmonary tuberculosis is lower than that in pulmonary tuberculosis.

In malnourished states, immune depression is found at the humoral and cellular levels, and is characterized by altered chemotaxis and phagocytosis, decreased serum albumin levels $(<3.5 \mathrm{~g} / \mathrm{dL})$ and decreased total lymphocyte counts $\left(<1,500-2,000\right.$ cells $\left./ \mathrm{mm}^{3}\right)$. Protein malnutrition results in a decreased number of circulating T-cells with impaired production of cytokines [17,18]. HIV patients' nutritional states in the late stage of the disease grow worse from the uncontrollable diarrhea $[17,18]$.

Immune system in the elderly patients is in general weakened, and reactivation of the latent tuberculosis is often reported [15,19].

Immune system compromise in diabetic patient has been associated with defects in humoral and cellular immune response. Humoral defects include deficits in complement protein $\mathrm{C} 3$ and $\mathrm{C} 4, \mathrm{C} 1$ inhibitor and alterations in antibody production in response to antigen. Cellular 
deficits have a more complex mechanism and involve alterations in cytokine signaling through tumour necrosis factor (TNF), interleukin (IL)-1 $\beta$, IL-2, IL-6, IL-8 and insulin growth factor (IGF)-2 and direct effects on T-cells and their relative populations [19-21].

HIV/AIDs predisposes patients to fungal and tuberculous infections because of defects in the neutrophils, a decreased cell counts and leucocyte dysfunction. In tuberculous infection, CD4+ lymphocyte (helper-inducer T-cell) count falls below 200 cells $/ \mathrm{mm}^{3}$, and in MAC infection CD4+ lymphocyte count falls below 100 cells $/ \mathrm{mm}$ $[2,17,18]$.

Anti-rheumatic agents (corticosteroids, methotrexate, chemotherapeutic and biologic agents) exert their effects through humoral and cellular levels of immune system and marrow suppression. TNF- $\alpha$ blockers inhibit lymphocytic and macrophage activity $[16,20,21]$.

\section{Clinical Manifestations}

At its active stage, symptoms of tuberculous spondylitis are often insidious. Common symptoms are malaise, loss of appetite and weight, and night sweat. The involved spine is stiff and painful on movement with a localized humpback. Back muscle spasms are present. Occasionally, patients may have night-cries during sleep, as the relaxation of muscle spasms allows for movement between the inflamed surfaces. Cold abscess and/or sinus may be present. In the early stages of disease, some of these symptoms and signs may be absent. On palpation, a small gibbus may be detected. Rarely, neurological deficits may present as the first symptom.

Despite the recent advances in the diagnosis and management of spinal tuberculosis, there remain a significant number of issues that up to now have received little attention in spite of its significant clinical importance [5]. This paper seeks to address these issues in diagnosis and management of spinal tuberculosis, in particular focusing on the newer diagnostic techniques including nonculture laboratory methods, hyperbaric oxygen therapy, immunosuppressant drug use, surgical options for the spinal deformity and associated neural involvement, instrumentation and implant removal time and the fate of the instrument-immobilized joints.

\section{Diagnosis}

The insidious onset, lack of early constitutional symptoms and local signs of spinal tuberculosis make early diagnosis difficult. With the increasing incidence of all tuberculosis types across the globe, physicians and surgeons must exercise a high index of suspicion to achieve early diagnosis.

Clinicians typically rely on a battery of means in diagnosing spinal tuberculosis. These include observation and investigation of clinical signs and symptoms and the use of various imaging techniques, smear and/or culture, metabolic product detection (interferon- $\gamma[$ IFN- $\gamma]$ test), polymerase chain reaction (PCR) and histological study. Diagnosis may take days to weeks, and involves expensive, invasive and complex procedures $[1,2,4,5]$. There is currently no single diagnostic method that can detect all tuberculosis types and cases.

\section{Tuberculin Skin Test}

The tuberculin skin test (TST) has inherent limitations of sensitivity and specificity. Even in high tuberculosisburden areas, approximately $20 \%$ of individuals show negative to TST throughout life, despite repeated exposure to the tubercle bacilli. Additionally the sensitivity decreases in immuno-compromised patients for whom accurate diagnosis of latent tuberculosis infection is essential. In terms of specificity, TST is influenced by Bacillus Calmette-Guérin (BCG) vaccination and nontuberculous mycobacterial infection $[2,5]$.

\section{Imaging}

Imaging techniques such as simple radiographs, bone scan, computed tomography (CT) and magnetic resonance imaging (MRI) are useful but not diagnostic. For example, when disc and/or end-plate destruction with surrounding soft tissue swelling is observed on simple radiographs, spine infection should be suspected.

Diffusion-weighted MRI has been found to have limited usefulness for differentiating spinal infection and malignancy.

\section{Laboratory}

Laboratory aids to diagnosis include: 1) complete blood 
counts including total lymphocyte and CD4 lymphocyte (helper-inducer T-cell) counts; 2) ESR and CRP; smear and/or culture; histology; detection of specific antigen [22]; metabolic products; 3) patient's antibody response and detection of antibody to M. tuberculosis; 4) DNA sequence polymerase-chain reaction (PCR) of M. tuberculosis.

The diagnostic gold standard has traditionally been the isolation of M. tuberculosis by smear and/or culture from clinical samples (e.g., aspirates and tissue specimens) and the typical histology. However, the tubercle bacilli are difficult to culture due to the fastidious growth requirements and the slow rate of growth. Hence there is a need for the development of varied laboratory methods.

There are three diagnostic non-culture laboratory tests: 1) immunological tests (antigen and antibody [22]); 2) metabolic product detection such as extracorporeal IFN- $\gamma$ test; and 3) amplification of DNA of M. tuberculosis by PCR [23].

The AMRAD ICT tuberculosis test (in vitro immunological tool for the detection of antibodies to M. tuberculosis in white blood cells) reliably detects tuberculosis in whole blood, enabling rapid and accurate diagnosis at the point of care $[2,5]$.

Lymphocytes secrete IFN- $\gamma$ when T-lymphocytes are exposed to tubercle bacilli, making extracorporeal IFN- $\gamma$ test for latent tuberculosis particularly noteworthy. Two ex-vivo IFN- $\gamma$ assay kits have been marketed recently: Quanti-FERON-TB Gold (QFT-G, Cellestis Limited, Carnegie Victoria, Australia); and T-SPOT, TB (T-SPOT, Oxford Immunotec, Oxford, UK [2,5]).

The former uses the ELISA technique, and T-SPOT uses enzyme-linked immunosorbent spot (ELISPOT) technique. Both use M. tuberculosis-specific antigens, 6 $\mathrm{kDa}$ early secretory antigenic target (ESAT-6) and $10 \mathrm{kDa}$ culture filtrate protein (CFP-10), as stimulants. As BCG does not contain ESAT- 6 and CFP-10, these assays are more specific than TST in the diagnosis of tuberculosis infection. They have variable sensitivity, as dependent on host factors and methods used. There are reports that T-SPOT is more sensitive than TST in immuno-compromised subjects.

These assays detect tuberculosis infection, but they cannot differentiate between latent tuberculosis infection and active tuberculosis. Therefore, when the TST and IFN- $\gamma$ assay results are positive, differentiation of latent infection and active tuberculosis should be carried out on the basis of the clinical history, chest and skeletal imaging studies and tissue studies.

The other major non-culture test is the molecular diagnostic test, PCR, which amplifies the DNA of M. tuberculosis and provides results within hours. This allows for effective and prompt treatment, which in turn improves prognosis. A primer pair targeting a 123 base pair (bp) segment of the repetitive sequence IS6110 of the M. tuberculosis complex is used, which covers M. tuberculosis, M. africanum, M. bovis, M. canetti and M. microti. This technique represents an exciting development. It has been used as a marker to monitor responses to treatment and has been shown to provide rapid information on drug resistance and clonality in epidemiological investigation of outbreaks.

Accurate diagnosis should be made at initial presentation to ensure proper treatment and the management decision must be based on the different treatment goals of individual patients.

\section{Classification Based on Staging of Spinal Tuberculosis}

Disease staging must be of practical use in management. There are no universally accepted staging systems, though several staging systems have been reported. Kumar [6], Moon [5], Mehta and Bhojraj [24], and Oguz et al. [25] proposed their therapeutic classification of the spinal tuberculosis based on the clinical stage of the disease processes. However, they could not satisfy the clinicians' requirement in management of tuberculous lesions of any spinal level. The focus of attention was on the aesthetic view points, rather than on the effect of residual spinal deformity on the remaining mobile segments, including the parafusion segments.

Terms such as early and late, early and advanced, kyphotic and non-kyphotic and stage I, II, and III are used in staging the disease. The vertebral body loss is expressed as a percentage and severity of the deformity. A more satisfactory system is still awaited.

\section{Myobacterium tuberculosis and Its Behavioral Characteristics}

Tubercle bacillus is a tiny thin rod shaped, non-motile, non-spore-forming obligate aerobic bacteria without pili for adherence, measuring $3 \mu \mathrm{m}$ in length and $0.5 \mu \mathrm{m}$ in 
width. Its generation time is 12 hours. M. tuberculosis gets into the phagocyte by being engulfed as phagosome, and starts its intracellular existence. If phagocyte fails to fuse with lysosome, engulfed bacillus cannot be killed by digestion, and continues to survive and divide inside the phagocyte.

Before initiation of any treatment, a good understanding of the nature of spinal tuberculosis is vital. Knowledge of the bacterial response to chemotherapy and biomaterial implantation is important. Bony tuberculosis has a low bacillary load (i.e., smaller population). M. tuberculosis presents in planktonic but not sessile form as seen in pyogenic bacteria, and occasionally becomes dormant and produces minimal adhesion molecules and slime. Because of the relatively slow replication rate of $M$. tuberculosis, antituberculous chemotherapy given intermittently is as effective as that given daily $[1,2,5]$.

Cell wall is chemically constituted by the several different mycolic acid compounds; 1) Mycocide is a compound of mycolic acid (a long fatty acid) bound to carbohydrate forming a glycolipids, 2) Cord factor (formed by the union of 2 mycolic acid with a disaccharide [trelalose]) and found only in virulent strains. It releases the tumor necrosis factor (TNF or cachectin) which causes rapid weight losss. 3) Sulfatide (sulfate attached to disaccharide) inhibits phagolysosomal fusion. 4) Wax D is a complicated mycoside which activates the protective cellular immune system.

Tubercle bacilli have very thick waxy envelope containing mycolic acids and phenolic glycolipids. The waxy coat impedes the entry of nutrient through poorly developed and scarce porins and thus limits growth rate, but it also protect the bacteria from host defenses and antibiotics. For this reason, the cure of tuberculosis requires an exceptionally long course of antibiotic therapy. Dormant bacilli in the latent tuberculosis are activated by various factors (Fig.1).

There were concerns that the use of the biomaterials in the tuberculous focus would hinder the healing of the tuberculosis, or cause arrested or quiescent infection to flare up, until implants such as titanium cage, screws and rods, polyvinyl propyrene and stainless steel or ceramic cups in the tuberculous focus were removed, but those biomaterials were found to be harmless [26-30].

M. tuberculosis has little tendency to adhere to implants which can be safely used in tuberculous lesions. However in HIV positive patients, the use of biomaterials in the infected foci is inappropriate, because they are susceptible

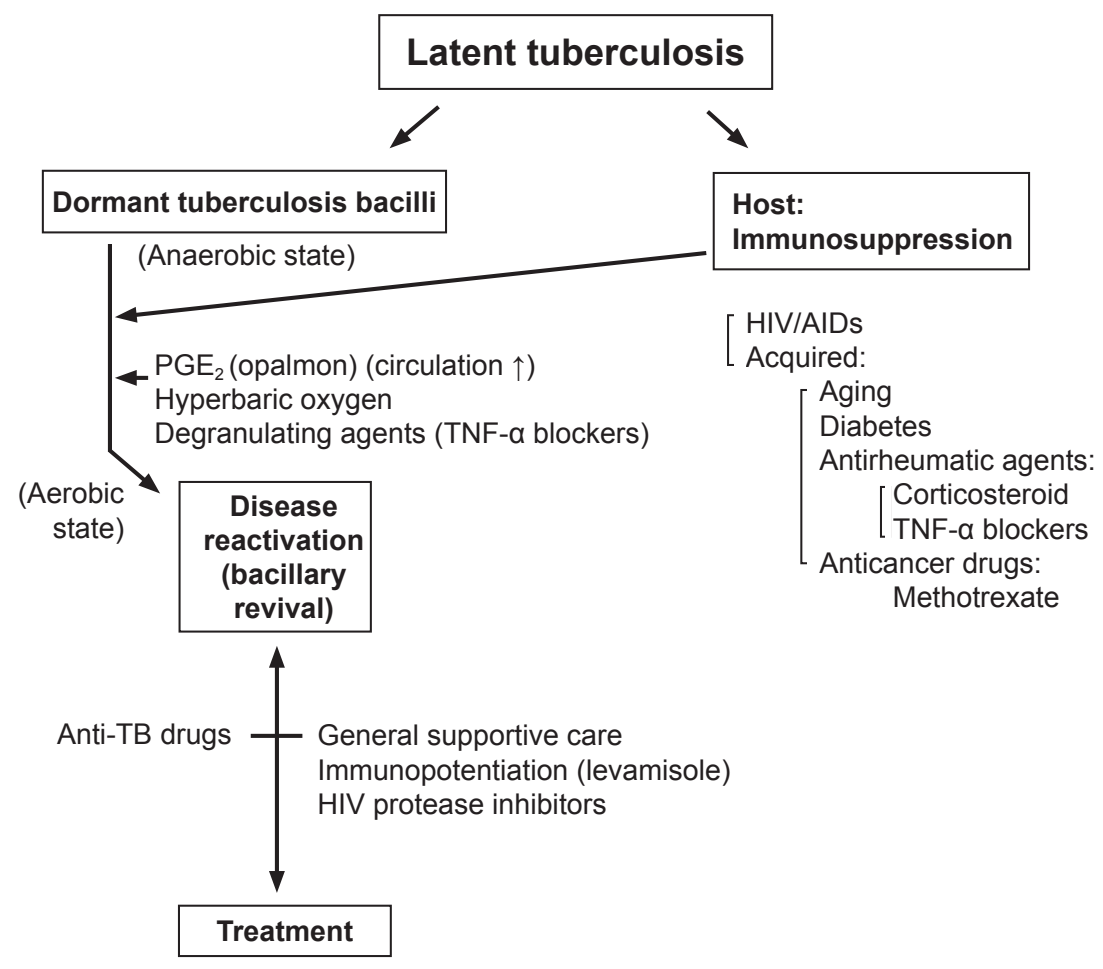

Fig. 1. Causes of reactivation of latent tuberculosis and management. HIV, human immunodeficiency virus; AIDs, acquired immunodeficiency syndrome; TNF, tumour necrosis factor tumour necrosis factor; TB, tuberculosis. 
Table 1. First and second line antituberculous agents

\begin{tabular}{ll}
\hline First-line agents & Second-line agents \\
\hline Isoniazid (H) & Amikacin (AMK) \\
\hline Rifampicin (rifampin) (R) & Capreomycin (CPM) \\
\hline Pyrazinamide (Z) & Ciprofloxacin (Cip) \\
\hline Ethambutol (E) & Cycloserine (CS) \\
& Ethionamide (Eth) \\
& Kanamycin (K) \\
& Ofloxacin \\
& P-aminosalicylic acid (PAS) \\
& Streptomycin (S) \\
& Rifabutin (Rif) \\
& Clarithromycin (Cla)
\end{tabular}

MAC, Mycobacterium avium complex.

to a variety of other opportunistic infections $[5,17,18]$.

Additionally bacteria adhere less to titanium than stainless steel, because titanium has a less electrochemically active oxide surface compared to stainless steel, which has a higher surface free energy $(>40 \mathrm{mN} / \mathrm{m})$ and hence more available ligands for binding with cells, both bacterial and tissue competing for the biomaterial surface [26-29,31,32]. It follows that stainless steel implants should not be used for spinal stabilization, as it has less resistibility to bacterial adhesion and infections.

\section{Management}

The treatment goals of spinal tuberculosis are primarily to eradicate the infection and to save life. Secondly the goals are: to provide stability for the affected spine; to meet the patient's aesthetic demand by preventing and/or correcting spinal deformities (not only for aesthetic purpose but also for reducing the parafusion segment disease); and to prevent or treat paralysis. The ideal management would be one that meets all above goals.

The management of spinal tuberculosis consists of supportive care, chemotherapy and surgery. Surgical measures include: cold abscess drainage; focal debridement of the tuberculous lesion and/or anterior fusion; decompression surgery including costotransversectomy and anterior radical surgery [33]; a two-stage procedure of posterior instrumentation [34] and anterior radical surgery [35]; a combined procedure of anterior radical surgery and anterior instrumentation; and corrective spinal osteotomy for healed rigid kyphosis [2,5]. However, surgery alone can-
Table 2. Accepted antituberculous regimens

Three drug regimens
Davies (1996) [19]: 2 HRZ 10 HR (12 mo)
Drug resistance case: 2 S(E) HRZ
Moon et al. (1987,1997,2003) [40,1,41]: 12 RHE(Z) (12 mo)
Upadhyay et al. (1993) [39]: AIF
3 HPaS, 3 HPa (6 mo)
3 HPaS 6 HPa (9 mo)
Four drug regimens:
Yilmaz et al. (1999) [27]: 2 SRHZ 7 RH (9 mo)
Mehta and Bhojraj (2001) [24]: 2 RHEZ 4 RHE 6 RH (12 mo)
Govender and Kumar (2001) [18]: 12 RHZE (12 mo)
Sundararaj et al. (2003) [28]: 4 RHEZ, 14 RHE (18 mo)
H, INH (isoniazid); R, rifampin; Z, pyrazinamide; E, ethambutol; Pa,
PAS (para-aminosalicyic acid); S, streptomycin; AlF, anterior interbody
fusion.

not cure the active disease, and effective antituberculous chemotherapy is necessary. Although the final fusion of the affected vertebral segment is an important part of the treatment, it does not necessarily accompany the spontaneous healing process.

Recently the current author found an important fact that spinal malalignment of the fused segment at the time of the disease cure in adults is a contributing factor increasing the incidence of parafusion segment disease [31]. Therefore maintenance and/or restoration of normal spinal alignment at the time of the disease cure is strongly recommended.

\section{Nutritional Therapy}

Hyperalimentation may be necessary to restore patients from a physically debilitated pre-therapy state to the premorbid nutritional status. The goal should be to achieve a serum albumin level $>3 \mathrm{~g} / \mathrm{dL}$, an absolute lymphocyte count $>800 / \mathrm{mm}^{3}$ and a 24 hours urine creatinine excretion $>10.5 \mathrm{mg}$ in men and $>5.8 \mathrm{mg}$ in women $[5,17,18]$.

\section{Chemotherapy}

When a patient presents early with minimal to moderate bony involvement that does not seem to cause noticeable deformity, conservative chemotherapy alone is indicated. Chemotherapy can be given on an ambulatory basis without bracing (Tables 1-3) [36-39]. Effective institution 
Table 3. Proposed chemotherapy formula for the drug-resistant $M$. tuberculosis

\begin{tabular}{llll}
\multicolumn{1}{c}{ Resistant agents } & \multicolumn{1}{c}{ Suggested regimens } & \multicolumn{1}{c}{$\begin{array}{c}\text { Duration of therapy } \\
(\mathrm{mo})\end{array}$} & \multicolumn{1}{c}{ Comments } \\
\hline INH, SM, PZA & RMP, PZA, EMB, AMK & $6-9$ & Estimated 100\% response rate and $<5 \%$ relapse rate \\
\hline INH, EMB $( \pm$ SM) & RMP, PZA, OFL/CIP, AMK (Pry) & $6-9$ & Efficacy should be comparable to the above regimen \\
\hline INH, RMP $( \pm$ SM) & PZA, EMB, OFL/CIP, AMK & $18-24$ & Consider surgery \\
INH, RMP, EMB $( \pm S M)$ & PZA, OFL/CIP, AMK, Plus 2 & 24 mo after conversion & Consider surgery \\
INH, RMP, PZA $( \pm S M)$ & EMB, OFL/CIP, AMK, Plus 2 & 24 mo after conversion & Consider surgery \\
INH, RMP, PZA, EMB $( \pm S M)$ & OFL/CIP, AMK, Plus 3 & 24 mo after conversion & Surgery, if possible \\
\hline
\end{tabular}

INH, isoniazid; SM, streptomycin; PZA, pyrazinamide; RMP, rifampin; EMB, ethambutol; AMK, amikacin; OFL, ofloxacin; CIP, ciprofloxacin; Pyr, pyronins.

Table 4. Advantages and disadvantages of chemotherapy alone

\begin{tabular}{ll} 
Advantages & \\
Arrest and/or cure tuberculosis & Cannot shorten the duration of chemotherapy \\
Can be done on an ambulatory basis & Longer time to cure the disease \\
\hline Cost-effective & Cannot meet aesthetic demands \\
\hline Expected spontaneous growth correction of the pediatric spinal deformity & Delayed and/or less neurological recovery \\
\hline Can avoid surgical complications and residuals & \\
\hline
\end{tabular}

of chemotherapy and good general supportive care at an early stage of disease are the key to early eradication and minimal sequelae. The advantages and disadvantages of conservative treatment alone are given in Table 4.

Studies have shown that conservative treatment at an early stage is beneficial in preventing residual kyphosis. Proper selection of candidates is based on the estimated residual kyphosis after chemotherapy. This can be calculated using the following formula [12]:

Final kyphosis $=5.5^{\circ}+\left[30.5^{\circ} \times\right.$ degree of vertebral body destruction in 10 grades (0.1-1.0).

As yet, there is no standardized anti-tuberculosis chemotherapy regimen, though several formulae have been recommended (Table 2). Some clinicians support the use of a short-term triple chemotherapy $[37,38]$, while others advocate four-drug regimens [2,5]. First-line therapy for M. tuberculosis should include a combination of isoniazid, rifampin, pyrazinamide and either ethambutol or streptomycin (Table 1). Susceptibility testing should be conducted in all isolates and the regimen adjusted accordingly to include at least two agents that the isolate is sensitive to.

Nonsteroidal anti-inflammatory drugs may be useful in early disease to prevent lesions caused by nonspecific synovial inflammation and to inhibit or minimize bone resorption caused by prostaglandin-mediated inflammation $[1,2,5,13]$. Atypical organisms such as Mycobacterium avium intercellularae complex (MAC) and M. kansasii may require additional antibiotics. For the treatment of $M A C$, a three-drug regimen including rifabutin, ethambutol and clarithromycin is the regimen of choice (Table 1). Multiple-drug therapy is more effective than monotherapy for the treatment of disseminated MAC. Regimens targeting $M A C$ are suppressive rather than curative, and thus therapy must be continued indefinitely (Table 1).

For HIV-negative patients, treatment should be started with at least three agents (isoniazid, rifampicin, pyrazinamide) or, if resistance is suspected, four (the above plus ethambutol) or more (Table 3). Despite diminished cell-mediated immunity, HIV-infected patients usually respond well to accepted antituberculosis regimens, provided that it is commenced promptly, the patient is compliant and the organism is not resistant. Isoniazid chemoprophylaxis is recommended for HIV-infected individuals with a positive tuberculin skin test, and for those with a history of exposure to an individual with active tuberculosis, regardless of the tuberculin skin test results [5].

There are no clear-cut definitions of good (or rapid) response, poor (or slow) response and non-response. The 
Table 5. Advantages of surgical treatment

Early healing by extirpation of the infected focus

Histological confirmation of disease activity

Shortening of chemotherapy

Reduction of late-recurrence rates

Correction and/or prevention of deformity

Early effective neurological recovery

To meet the patient aesthetic demands

recommended observation period for drug response differs between non-paralytics and paralytics. In non-paralytics, a 6- to-8-week assessment (maximum 3 months) is recommended, whereas in paralytics the assessment should take 3 to 4 weeks. However, labeling the patient as a nonresponder on the basis of an arbitrary assessment period may not be justified. That is, exact timing of drug response assessment in patients with or without neurologic deficit remains a matter of debate. Table 3 gives recommendations for managing resistant tuberculosis. The author suggests that treatment should last at least 12 months, although others consider 9 months sufficient to achieve a positive result and do not recommend longterm suppressive therapy after 9 months of chemotherapy [36-40].

For rheumatoid arthritis and related disease patients with latent tuberculous infection, anti-rheumatic agents such as anti-TNF- $\alpha$ treatment should be started at least after 3 weeks of anti-tuberculous chemotherapy. For rheumatoid patients with active tuberculosis, TNF- $a$ agent therapy should be started at least after 2 months of anti-tuberculous chemotherapy [5].

As most cases of tuberculosis after biologic therapy represent reactivation rather than a new onset infection, screening before TNF- $\alpha$ blocker therapy is critical to prevent such a relapse. However, it is a costly process. It is reminded that during anti-TNF- $\alpha$ therapy postoperative rigor or fever, other bacterial infection must be excluded.

\section{Surgical Treatment}

Chemotherapy alone, nevertheless, cannot correct problems arising from bone and joint destruction. Therefore, despite the availability of effective conservative treatments, surgical procedures still assume an important role in the management of spinal tuberculosis. Four conditions of spinal tuberculosis, namely cold abscess, tu- berculous lesion, paraplegia and spinal deformity, can be managed by various surgical procedures when indicated. The advantages of surgical treatment are given in Table 5 . Acceptable degree of residual kyphosis is also a topic of great controversy related with aesthetic and biomechanical aspect.

\section{Abscess Drainage}

In most cases, small and medium-size cold abscesses rapidly resolve spontaneously with chemotherapy alone. In the author's series, evacuation of abscesses did not alter patients' general condition, and very often it would result in a draining sinus from which drainage persisted for some time. Therefore drainage is not recommended as a routine practice even for large abscesses.

\section{Focal Debridement}

Focal debridement is rarely indicated nowadays, because evidence suggests it does not improve healing nor prevent the kyphosis from progressing [33]. Radical extirpation of the infected focus may also damage the remaining growth plates in children $[2,5,38]$. Although the procedure is simple, it carries a small risk for a number of complications such as dura tear, cord injury and injury to nerve roots, autonomous nerves and peripheral nerves.

\section{Anterior Radical Surgery (Anterior Debridement and Anterior Fusion)}

Bone grafting after anterior radical debridement is safe in the treatment of spinal tuberculosis even when drainage is present. Anterior radical surgery was once advocated as the treatment of choice for spinal tuberculosis [33,37]. However, it later became clear that the only advantage of anterior fusion is a certain extent of deformity correction and a decreased tendency for deformity progression. The author's experience suggests that, because of graft failure, the procedure is not always successful in preventing kyphosis progression and/or correcting pre-existing kyphosis.

Literature on the treatment of tuberculous kyphoscoliosis and scoliosis is scarce. With non-instrumented anterior radical surgery alone, even non-rigid deformities are difficult to correct, and the corrections, if any, are difficult to maintain. In rigid deformities, this is even more 
challenging and the complication rate is relatively high. When anterior surgery is used to treat nonrigid pure tuberculous scoliosis, some overcorrection is recommended to balance out the loss of correction during the fusion process. However, in cases of non-graft failure and absorption or graft bed failure, there may not be any loss of correction, and iatrogenic scoliosis in the opposite direction may occur. When this type of excessive scoliotic deformity occurs at L4 to L5 as a result of a rhomboid graft, clinically compensatory pelvic tilt may result. Therefore, care should be taken to avoid inducing excessive coronal deformity iatrogenically by the anterior intercorporal graft. If surgical treatment is indicated in tuberculous scoliosis, combined procedures such as posterior or anterior instrumented stabilization after anterior corrective radical surgery are preferred [5].

Spontaneous fusion of adjacent segment happens infrequently following anterior cervical spine surgery in children.

\section{Cord Injury by Graft}

Cord injury may occur during radical surgery, though the incidence is very low. Only two out of 764 anterior procedures done in the author's unit has resulted in a cord bruise from the graft placement into the spinal canal. Utmost attention should be paid in positioning the grafts between the vertebral bodies.

\section{Stabilizing Instrumentation}

Corrective and stabilizing spinal surgery is most effective in active progressive kyphosis and nonrigid kyphosis. However, posterior instrumentation surgery is not recommended in early tuberculosis, because the disease can be cured with conservative treatment without significant residual kyphosis.

In anterior column destruction with kyphosis, posterior instrument-aided correction of kyphosis and stabilization will produce a bone gap in the anterior column. This should be reconstructed by anterior strut graft surgery. Failure to do so may result in corporal re-collapse and/or instrumentation failure.

In children with both anterior and posterior spinal elements involved, the affected segment becomes unstable, causing spinal deformity and an increased risk of paraplegia. Prophylactic stabilization should be performed if translational instability is suspected at the level of the lesion, particularly in children $[1,2,5]$.

\section{Posterior Instrumentation and Anterior Radical Surgery}

The author's clinical experience suggests that it is unwise to rely solely on the anterior strut graft to correct the spinal deformity and to maintain the correction without graft collapse. In view of this, the author sought and adopted since 1981 a method of treatment that could prevent major spinal deformity-a combined two-stage procedure [7,42-44].

Patients presenting late with extensive, multisegment tuberculosis involving more than two vertebral bodies and kyphosis are suitable candidates for the two-stage procedure, which includes posterior stabilizing and corrective instrumental surgery together with anterior radical surgery, under the cover of chemotherapy. Corrective and stabilizing spinal surgery is most effective in active disease with nonrigid spinal deformity.

Instrument-aided multi-segment posterior stabilization of the spine using long rods is more reliable in correcting and stabilizing flexible spinal deformity without graft failure. Stabilization with posterior instrumentation has several advantages, and include arresting the disease early and bringing about early fusion, correcting the deformity and maintaining the corrected alignment.

With the combined two-stage procedure, the collapsed vertebra can regain its height. Also the width and/or the extent of anterior interbody fusion can be minimized and the adjacent unaffected vertebrae preserved. The remaining vertebral body can be used as a graft bed. No graft fracture or absorption has been observed in the author's series. Furthermore, the procedure can shorten the postoperative bed-rest period [43].

However, long rodding immobilizes also the normal mobile segments, and may later cause these segments to stiffen and degenerate. Therefore, the author normally removes the rods after solid intercorporal fusion at about 9 months postoperation to remobilize these segments. In children, care should be taken not to damage the remaining growth plate during the anterior curettage surgery, and combined posterior instrumentation and posterior fusion is recommended to prevent and/or correct kyphosis $[2,5,11,33]$. 


\section{Residual Spinal Deformity and Parafusion Segment Disease}

Maintenance of normal spinal alignment of the diseased segment and also the restoration of deformed segment to normal alignment are essential to reduce the ill-effect on the proximal and distal mobile segments. Since 2001 the current author has strongly recommended maximally to minimize the residual kyphosis less than $15^{\circ}$ in case of mono-segment involvement and less than $10^{\circ}$ in case of bisegment involvement [5,31,32].

\section{Posterior Fixation Failure and Increase of Deformity}

Failure of posterior stabilization because of rod and/or screw loosening and/or fracture of posterior rods is rare, but if it occurs it is a formidable complication, particularly in children with rapidly progressing spinal deformity.

\section{Anterior Radical Surgery and Anterior Instrumentation}

Controversies exist regarding the use of biomaterial in the infected focus to immobilize the diseased segments internally. Generally, implantation of biomaterial in the infected focus is contraindicated, because the biomaterial may serve as nest for pyogenic bacteria. However, this is not the case in bone tuberculosis. Bacteria normally produce adhesion molecules to adhere to and survive on the pits of the synthetic surface. M. tuberculosis, however, divides very slowly with minimal glycocalyx slime production and exists in a planktonic form, which responds well to chemotherapy. This is thought to be the reason why the fixation or reconstruction biomaterial in the tuberculous focus would not cause biomaterial-centered infections. The use of in-situ anterior instrumentation in skeletal tuberculosis is therefore acceptable: it provides effective mechanical stability until the tuberculosis heals and does not hinder the healing process $[1,2,5,45,46]$.

\section{Corrective Surgical Procedures for Severe Rigid Kyphosis}

In the past if it was only for cosmetic reasons, corrective surgery was not recommended in healed severe kyphotic or kyphoscoliotic tuberculous spine with normal neurol- ogy because of high complication rates of neurological injury, particularly above T11.

Instrument-aided posterior corrective/stabilizing surgery and anterior reconstruction surgery are most effective in the management of active progressive kyphosis and established residual non-rigid kyphosis [43].

Recently non-paralytic young patients with severe rigid kyphosis tend to have very high aesthetic demands. This has led to the development of safe and effective corrective surgical procedures for the unsightly kyphosis, but each patient must be considered individually.

In healed severe tuberculous kyphosis at cord level, the bony anatomy is severely deformed, and the cord in this area is ischemic, deformed and less mobile. It is often trapped in the deformed, narrow, hypoplastic canal and is adherent to the dura $[5,46]$.

There are several possible surgical procedures, ranging from single-stage to multistage procedures for rigid tuberculous kyphosis, including posterior closing wedge osteotomy, decancellation procedure and hemi- or total spondylectomy as a single stage operative procedure $[1,2,5,41,47]$. Long segment stabilization and fusion are essential in this surgery. However, it can cause the adjacent joint disease in adults later in life. As such case continuous observation should be practiced [48].

Any decision to operate must weigh the high complication and re-operation rates against anticipated improvement. An informed consent discussion should include the patient and his or her support group. Risks and complications must be fully understood; and the patient will ultimately choose balancing between those risks and his/ her quality of life. The surgeon should offer options and probability, but it is the patient who must ultimately bear the consequences of the decision $[5,41,49]$.

\section{Spinal Column Shortening}

The ideal size of the longitudinal spondylectomy in the correction of the spinal deformity in relation with the cord function has rarely been discussed, and there is no consensus. In the normal spine, cord length, spinal canal and anterior spinal column length are equal, while in the idiopathic adolescent scoliosis, the anterior column length is longer than the canal length. Therefore, if the scoliotic spinal column is corrected by longitudinal distraction alone, the cord will be stretched, and may result in paralysis. When total en-bloc spondylectomy is per- 
formed for a spine tumor, the preoperative relationship of the cord and spinal column length should be maintained. Sometimes the spinal column can be lengthened or shortened at the time of anterior column reconstruction and instrumented stabilization. In the former the cord will be stretched and in the latter the cord will be axially compressed which results in cord ischemia leading to paralysis.

Kawahara et al. [50] reported that the dural kinking was not observed in any patients undergoing a column shortening procedure, with $22.2 \%(7 \mathrm{~mm} \pm 4-10 \mathrm{~mm}$ ) of the corpectomy defect in 40 patients. Postoperatively neurological degradation was observed in none. They concluded that $20 \%$ column shortening in spinal tumor surgery might be safe.

Kobayashi et al. [51] reported the results in dogs of longitudinal column shortening surgery. Up to $7.2 \mathrm{~mm}$ shortening produced no morphological change in the dura mater or spinal cord. If the shortening was between 7.2 and $12.5 \mathrm{~mm}$, the cord remained straight in spite of 'ruffling' of the dura mater. Above $12.5 \mathrm{~mm}$ of shortening, the cord became kinked. Abnormal evoked potentials were recorded with column shortening of 15 and 20 $\mathrm{mm}$, causing incomplete paralysis of the hind limbs in 1 of 3 and 3 of 4 dogs, respectively. With column shortening of $5,10,15$, and $20 \mathrm{~mm}$, cord blood flow changed to $146 \%, 160 \%, 102 \%$ and $93 \%$ of the preoperative blood flow, respectively. Column shortening less than $15 \mathrm{~mm}$ significantly increased the diameter of the anterior spinal artery, while shortening over $15 \mathrm{~mm}$ decreased the anterior flow. They concluded that the safety limit for column shortening was $12.5 \mathrm{~mm}$ (62.5\%).

Tanaka et al. [52] have reported their results of the cranial half-shortening spondylectomy $(14-23 \mathrm{~mm}$; mean, $20 \mathrm{~mm}$ ) of the L1 body for adult patients with tethered cord syndrome. There were symptomatic improvements after spinal column shortening. The procedure was found to be safe and effective, but they did not discuss spondylectomy size.

\section{Tuberculous Kyphosis in Children}

In children spontaneous intercorporeal fusion rarely occurs during chemotherapy $[1,2,5,12,30,40,53]$. It occurs later after the cure of the tuberculosis, at lower rates than in adults. This has not been satisfactorily explained. Study of BMP and anti-BMP factors in the tissue around the tuberculous lesion may clarify the cause.

In children, the deformity may correct spontaneously because of bone reformation from the remaining growth plates. Thus, the growth plates are especially important in children because of their capability of bone reformation of the destroyed vertebral body [11].

However in most children, there was a gradual increase of spinal deformity in spite of disease cure, when the growth plates were destroyed in either unfused or fused vertebrae. When the unfused segment was unstable, the deformity progressed more rapidly than that of the fused block vertebrae $[12,53]$.

In regards to the fused block vertebrae, there are two contradicting views on the effect of the vertebral block on the development of kyphosis during growth. According to Upadhyay et al. [39], the children who underwent radical surgery showed an overall correction which was virtually maintained up to final follow-up, and concluded that there is no disproportionate posterior spinal growth after anterior spinal fusion which contributes to the progression of deformity. On the contrary, Schulitz et al. [11] reported that they observed progressive kyphosis in the children with fused block vertebra. Rajasekaran and Shanmugasundaram [12] and Rajasekaran [53] reported that in children with unfused tuberculous kyphosis after cure of tuberculosis, there is a posterior longitudinal overgrowth that eventually causes facet joint subluxation with the greatest risk for spinal deformity. The current author supports the latter views.

The unsightly hunchback residual kyphosis can be distressing to patients, their parents and surgeons alike. Initially in the early 1970s, the author used posterior interspinous wiring and fusion. This was followed by interspinous wiring and cementation to stabilize the involved segment and to arrest the posterior spinal element growth. Both techniques failed because of loosening of wires and cement, and they were succeeded by posterior instrumentation $[7,42,44]$. Posterior instrumentation in the management of the pediatric tuberculous kyphosis can contribute to growth correction of the kyphosis by growth arrest of the posterior column when applied before age 10 to 11 years, though the effect of growth arrest may be different between the segmental stabilization of Lugue and pedicle screw fixation system [5,49].

In summary thus in children, posterior instrumentation is thought to be the best procedure with or without posterolateral fusion, utilizing several tether devices. 


\section{Tuberculosis in the Elderly}

The body's immune system is likely to have weakened from aging in the elderly. This may contribute to the emergence of tuberculosis in the previously infected individual. It may be that the rapid increase in life expectancy now occurring is resulting in an increase in the incidence of tuberculosis among those elderly people, because of both endogenous reactivation and exogenous infection $[15,19]$.

In general, there is an increase in reported adverse effects of drugs in elderly people. The presence of concomitant disease such as liver or renal failure may necessitate dosage adjustment of some of the treatment regimens $[5,15,19]$.

Streptomycin is excreted exclusively and ethambutol predominantly by the kidney. If either of these two drugs is to be given to patients with renal failure, the dosage should be adjusted accordingly. Serum concentration of streptomycin should not exceed $5 \mathrm{mg} / \mathrm{mL}$. If the patient is on dialysis, the dose should be given 5 hours beforehand. Ethembutol should be given in a dose of $25 \mathrm{mg} / \mathrm{kg}$, 3 times weekly, if the creatinine clearance is between 30 and $50 \mathrm{~mL} / \mathrm{min}$. Other main antituberculous medicines are hepatotoxic. For patients with alcoholism or for hepatitis B antigen carriers, isoniazid and rifampicin, together with one non-hepatotoxic drug should be given to ensure that the duration of treatment will not need to be prolonged.

\section{Pott's Paraplegia}

The three main causes of Pott's paraplegia are: 1) cord compression by abscess and granulation tissue; 2) cord compression by sequestrums and the posterior bony edge of the vertebral body at the level of the kyphosis; and 3) bony canal stenosis of the deformed spine above the level of the kyphosis [5,54].

Many factors affect recovery from Pott's paraplegia. These include: patient's general physical condition and age; condition of the spinal cord; level and number of vertebrae involved; severity of the spinal deformity (almost no recovery even after effective decompression surgery in patients with kyphosis of over $60^{\circ}$ ); duration and severity of the paraplegia; time to initiation of treatment; type of treatment and drug sensitivity.
There are two approaches to the treatment of Pott's paraplegia; conservative medical and radical $[41,54,55]$. Pott's paraplegia caused by abscess and granulation tissue in the early active stage can be managed effectively by chemotherapy alone. Surgical decompression is indicated when there is acute-onset paralysis with rapidly worsening neurology, bony cord compression, peridural fibrosis, and chronic compression within the narrow canal. The time frame for surgery clearly depends on the intensity of the neurologic deficit; and dense and progressive deficit needs surgical management much earlier $[24,54,56]$. All types of surgical complications can be encountered during anterior or posterior decompression surgery, though their incidence is low except in cases of rigid severe spinal deformity with distorted neural anatomy [5].

In one study, the neurological recovery rate in 33 Pott's paraparetics ( 7 healed, 26 active tuberculosis) with severe spinal deformity was investigated. All seven ( 2 children, 5 adults) paralytics with healed tuberculosis received surgery. Recovery was relatively good in the two children but poor in the five adults. Among the 26 cases with active disease, 10 ( 3 children, 7 adults) received chemotherapy alone, and 16 (3 children, 13 adults) underwent surgery. By chemotherapy alone, all three children recovered to a level near normal, and the seven adults also recovered relatively well. In the three surgically treated children, neurological deterioration was observed after surgery, though it later improved by one Frenkel's grade. In the thirteen surgically treated adults, a less degree of recovery was achieved. Thus, in spite of effective atraumatic decompression surgery, neurological recovery failure rate is high. The non-recovery was thought not to be due to definite surgical trauma but to the pre-existing myelopathy secondary to cord ischaemia, chronic peridural fibrosis and canal stenosis, especially in cases with kyphosis over $60^{\circ}$. The more severe the kyphosis, the worse the prognosis. Tuli [14] stated that a $60^{\circ}$ kyphosis seems to be the watershed for possible neurological recovery after decompression surgery.

Neurological recovery was relatively good in children and poor in adults. Although the severity of the paralysis varied between the conservative and surgical groups at the time of treatment, better recovery was achieved in the conservatively treated group and in children [41].

During surgery, there is always a risk of injuring the spinal cord, particularly with extensive anterior decompression at any level of the spine, if the normal landmarks are 
distorted by the disease. For this reason, meticulous control of hemorrhage is recommended during surgery to identify the dural sac when the anatomical landmarks are severely distorted. In the operative field, one can identify a nerve root and follow it centrally until the dura and the cord are safely identified. An alternative approach is to initiate the decompression over an unaffected area and proceed to the target area following the plane of the dura [5].

If neurological functions worsen in spite of chemotherapy in a Pott's paraplegic patient with severe spinal deformity, only the decompressive surgery is indicated to arrest the progression of paralysis and hopefully to restore normal neurology, though in the past some surgeons combined the corrective surgery at the time of decompression. There is only a small chance of neural recovery by surgery, and all patients should be informed of this. The greatest neurological risk is perhaps associated with the correction of a severe fixed kyphosis by osteotomy, and the patients and their family should be advised preoperatively that cord injury may complicate this procedure.

In Moon et al. [54]'s series of 67 paraplegic patients with minimal to moderate kyphosis, all 13 conservatively treated and $47(87.0 \%)$ of the 54 surgically treated patients recovered. The difference in the neurological recovery rates between the two groups was attributed to the difference in the stage of the disease at the time of treatment in addition to surgical complications [54].

In the current author's experience, posterior instrument-aided stabilizing surgery alone regardless of the disease stage and/or severity of deformity can hasten the neurological recovery without direct decompression surgery [5].

\section{Extent of Instrumentation and Timing of Implant Removal}

After corrective surgery of the severe deformity, multisegment-stabilization is inevitable including the 3 to 4 normal mobile segment, while for the monosegment lesion, short segment fixation is rather recommended because of early intercorporal fusion of the affected segment. Multi-segment instrumentation immobilizes normal mobile segments and may later cause these segments to stiffen and degenerate. Therefore the author normally removes the instrument after solid intercorporal fusion about 9 months postoperatively to remobilize these in- strumented segments, though there is no acceptable regimen for all the surgeons $[2,5,57]$.

Preservation of normal proximal and distal mobile segments, particularly in the cervical and lumbar spine, is strongly recommended to restore and/or maintain the near-normal spinal column functions.

In children with unacceptable residual kyphosis, posterior instruments should be left until maturity to maximize gradual growth correction of the deformity due to the tethering effect of the implants $[2,5]$.

\section{Conclusions}

Spinal tuberculosis is curable with the presently available chemo-therapeutic agents. With early detection and institution of chemotherapy and improved surgical techniques, patients with kyphosis are rarely seen in daily practice currently. Selection of the therapeutic measures to minimize the residual kyphosis in the highly mobile spinal segments after cure of the disease is stressed not only for aesthetic view point but also for reduction of incidence of parafusion segment disease. Paraplegic patients can be well managed with minimal residuals if early diagnosis and effective treatment are achieved.

\section{Conflict of Interest}

No potential conflict of interest relevant to this article was reported.

\section{References}

1. Moon MS. Tuberculosis of the spine: controversies and a new challenge. Spine (Phila Pa 1976) 1997;22:1791-7.

2. Moon MS. Managing tuberculosis of spine. Med Progr (Hong Kong) 2004;31:593-602.

3. China Tuberculosis Control Collaboration. The effect of tuberculosis control in China. Lancet 2004;364:417-22.

4. Moon MS. Development in the management of tuberculosis of the spine. Curr Orthop 2006;20:132-40.

5. Moon MS. Tuberculosis of spine: contemporary thoughts on current issues and perspective views. Current Orthopaedics 2007;21:364-79.

6. Kumar K. Tuberculosis of spine: natural history of disease and its judicious management. J Western Pac 
Orthop Assoc 1988;25:1-18.

7. Moon MS. Treatment of spinal infections. Commemorative issue. 3rd Spinal Section Congress, WPOA. J Western Pac Orthop Assoc 1983;20:7-11.

8. Beronius M, Bergman B, Andersson R. Vertebral osteomyelitis in Goteborg, Sweden: a retrospective study of patients during 1990-95. Scand J Infect Dis 2001;33:527-32.

9. Carragee EJ. Pyogenic vertebral osteomyelitis. J Bone Joint Surg Am 1997;79:874-80.

10. Chelsom J, Solberg CO. Vertebral osteomyelitis at a Norwegian university hospital 1987-97: clinical features, laboratory findings and outcome. Scand J Infect Dis 1998;30:147-51.

11. Schulitz KP, Kothe R, Leong JC, Wehling P. Growth changes of solidly fused kyphotic bloc after surgery for tuberculosis. Comparison of four procedures. Spine (Phila Pa 1976) 1997;22:1150-5.

12. Rajasekaran S, Shanmugasundaram TK. Prediction of the angle of gibbus deformity in tuberculosis of the spine. J Bone Joint Surg Am 1987;69:503-9.

13. Moon MS, Moon YW, Moon JL, Kim SS, Sun DH. Conservative treatment of tuberculosis of the lumbar and lumbosacral spine. Clin Orthop Relat Res 2002;(398):40-9.

14. Tuli SM. Severe kyphotic deformity in tuberculosis of the spine. Int Orthop 1995;19:327-31.

15. Maeda Y, Izawa K, Nabeshima T, Yonenobu K. Tuberculous spondylitis in elderly Japanese patients. J Orthop Sci 2008;13:16-20.

16. Cunningham ME, Girardi F, Papadopoulos EC, Cammisa FP. Spinal infections in patients with compromised immune systems. Clin Orthop Relat Res 2006;444:73-82.

17. Govender S, Parbhoo AH, Kumar KP, Annamalai K. Anterior spinal decompression in HIV-positive patients with tuberculosis. A prospective study. J Bone Joint Surg Br 2001;83:864-7.

18. Govender S, Kumar KP. Cortical allografts in spinal tuberculosis. Int Orthop 2003;27:244-8.

19. Davies PD. Tuberculosis in the elderly. Epidemiology and optimal management. Med Progr (Hong Kong) 1996;23:25-8.

20. Kim HA, Yoo CD, Baek HJ, et al. Mycobacterium tuberculosis infection in a corticosteroid-treated rheumatic disease patient population. Clin Exp Rheumatol 1998;16:9-13.

21. Genestier L, Paillot R, Fournel S, Ferraro C, Miossec P,
Revillard JP. Immunosuppressive properties of methotrexate: apoptosis and clonal deletion of activated peripheral T cells. J Clin Invest 1998;102:322-8.

22. Cole RA, Lu HM, Shi YZ, Wang J, De-Hua T, Zhou AT. Clinical evaluation of a rapid immunochromatographic assay based on the $38 \mathrm{kDa}$ antigen of Mycobacterium tuberculosis on patients with pulmonary tuberculosis in China. Tuber Lung Dis 1996;77:3638.

23. Brisson-Noel A, Gicquel B, Lecossier D, LevyFrebault V, Nassif X, Hance AJ. Rapid diagnosis of tuberculosis by amplification of mycobacterial DNA in clinical samples. Lancet 1989;2:1069-71.

24. Mehta JS, Bhojraj SY. Tuberculosis of the thoracic spine. A classification based on the selection of surgical strategies. J Bone Joint Surg Br 2001;83:859-63.

25. Oguz E, Sehirlioglu A, Altinmakas M, et al. A new classification and guide for surgical treatment of spinal tuberculosis. Int Orthop 2008;32:127-33.

26. Ha KY, Chung YG, Ryoo SJ. Adherence and biofilm formation of Staphylococcus epidermidis and Mycobacterium tuberculosis on various spinal infections. Spine (Phila Pa 1976) 2004;29:1-6.

27. Yilmaz C, Selek HY, Gurkan I, Erdemli B, Korkusuz Z. Anterior instrumentation for the treatment of spinal tuberculosis. J Bone Joint Surg Am 1999;81:1261-7.

28. Sundararaj GD, Behera S, Ravi V, Venkatesh K, Cherian VM, Lee V. Role of posterior stabilisation in the management of tuberculosis of the dorsal and lumbar spine. J Bone Joint Surg Br 2003;85:100-6.

29. Kim YY, Ko CU, Lee SW, Kwak BM. Replacement arthroplasty using the Charnley prosthesis in old tuberculosis of the hip. Int Orthop 1979;3:81-8.

30. Controlled trial of short-course regimens of chemotherapy in the ambulatory treatment of spinal tuberculosis. Results at three years of a study in Korea. Twelfth report of the Medical Research Council Working Party on Tuberculosis of the Spine. J Bone Joint Surg Br 1993;75:240-8.

31. Moon MS, Moon JL, Kim SS, Moon YW. Treatment of tuberculosis of the cervical spine: operative versus nonoperative. Clin Orthop Relat Res 2007;460:67-77.

32. Oga M, Arizono T, Takasita M, Sugioka Y. Evaluation of the risk of instrumentation as a foreign body in spinal tuberculosis. Clinical and biologic study. Spine (Phila Pa 1976) 1993;18:1890-4.

33. Hodgson AR, Stock FE. Anterior spine fusion for the treatment of tuberculosis of the spine: the opera- 
tive findings and results of treatment in the first 100 cases. J Bone Joint Surg Am 1960;42:295-310.

34. Zhao J, Lian XF, Hou TS, Ma H, Chen ZM. Anterior debridement and bone grafting of spinal tuberculosis with one-stage instrumentation anteriorly or posteriorly. Int Orthop 2007;31:859-63.

35. Moon MS. Combined posterior instrumentation and anterior interbody fusion for active tuberculous kyphosis of the thoracolumbar spine. Curr Orthop 1991;5:177-9.

36. Griffith DL. Short-course chemotherapy in the treatment of spinal tuberculosis. J Bone Joint Surg Br 1986;68:158-65.

37. Upadhyay SS, Saji MJ, Yau AC. Duration of antituberculosis chemotherapy in conjunction with radical surgery in the management of spinal tuberculosis. Spine (Phila Pa 1976) 1996;21:1898-903.

38. Moulding T, Dutt AK, Reichman LB. Fixed-dose combinations of antituberculous medications to prevent drug resistance. Ann Intern Med 1995;122:9514.

39. Upadhyay SS, Sell P, Saji MJ, Sell B, Yau AC, Leong JC. 17-year prospective study of surgical management of spinal tuberculosis in children. Hong Kong operation compared with debridement surgery for short- and long-term outcome of deformity. Spine (Phila Pa 1976) 1993;18:1704-11.

40. Moon MS, Kim I, Woo YK, Park YO. Conservative treatment of tuberculosis of the thoracic and lumbar spine in adults and children. Int Orthop 1987;11:31522.

41. Moon MS, Moon JL, Moon YW, et al. Pott's paraplegia in patients with severely deformed dorsal or dorsolumbar spines: treatment and prognosis. Spinal Cord 2003;41:164-71.

42. Moon MS, Rhee SK, Kang YK. Harrington rods in treatment of active spinal tuberculosis with kyphosis. J Western Pac Orthop Assoc 1986;23:53-8.

43. Klockner C, Valencia R. Sagittal alignment after anterior debridement and fusion with or without additional posterior instrumentation in the treatment of pyogenic and tuberculous spondylodiscitis. Spine (Phila Pa 1976) 2003;28:1036-42.

44. Moon MS, Woo YK, Lee KS, Ha KY, Kim SS, Sun DH. Posterior instrumentation and anterior interbody fusion for tuberculous kyphosis of dorsal and lumbar spines. Spine (Phila Pa 1976) 1995;20:1910-6.

45. Eysel P, Hopf C, Vogel I, Rompe JD. Primary stable anterior instrumentation or dorsoventral spondylodesis in spondylodiscitis? Results of a comparative study. Eur Spine J 1997;6:152-7.

46. Benli IT, Kaya A, Acaroglu E. Anterior instrumentation in tuberculous spondylitis: is it effective and safe? Clin Orthop Relat Res 2007;460:108-16.

47. Yau AC, Hsu LC, O’Brien JP, Hodgson AR. Tuberculous kyphosis: correction with spinal osteotomy, halo-pelvic distraction, and anterior and posterior fusion. J Bone Joint Surg Am 1974;56:1419-34.

48. Wong YW, Leong JC, Luk KD. Direct internal kyphectomy for severe angular tuberculous kyphosis. Clin Orthop Relat Res 2007;460:124-9.

49. Hosman AJ, Langeloo DD, de Kleuver M, Anderson PG, Veth RP, Slot GH. Analysis of the sagittal plane after surgical management for Scheuermann's disease: a view on overcorrection and the use of an anterior release. Spine (Phila Pa 1976) 2002;27:167-75.

50. Kawahara N, Tomita K, Murakami H, Akameru M, Hata T. Spinal shortening in total enbloc spondylectomy. Jpn Orthop Assoc Congress Book 2005;3A:P94.

51. Kobayashi T, Kawahara N, Murakami H, Fujita F, Tomita K. An experimental study on the influence of spinal shortening on the spinal cord. Jpn Orthop Assoc Congress Book 2005;3A:P9-1.

52. Tanaka Y, Kokubun S, Ozawa K, et al. Shortening spinal osteotomy for tethered cord syndrome. Jpn Orthop Assoc Congress Book 2005;3A:P9-2.

53. Rajasekaran $\mathrm{S}$. The natural history of post-tubercular kyphosis in children. Radiological signs which predict late increase in deformity. J Bone Joint Surg Br 2001;83:954-62.

54. Moon MS, Ha KY, Sun DH, Moon JL, Moon YW, Chung JH. Pott's paraplegia: 67 cases. Clin Orthop Relat Res 1996;(323):122-8.

55. Laheri VJ, Badhe NP, Dewnany GT. Single stage decompression, anterior interbody fusion and posterior instrumentation for tuberculous kyphosis of the dorso-lumbar spine. Spinal Cord 2001;39:429-36.

56. Jain AK, Sinha S. Evaluation of systems of grading of neurological deficit in tuberculosis of spine. Spinal Cord 2005;43:375-80.

57. Kahanovitz N, Arnoczky SP, Levine DB, Otis JP. The effects of internal fixation on the articular cartilage of unfused canine facet joint cartilage. Spine (Phila $\mathrm{Pa}$ 1976) $1984 ; 9: 268-72$. 